Voix et Images

voixetimages

\title{
Entrevue avec Fulgence Charpentier
}

\section{Marie-Pier Luneau et Pierre Hébert}

Volume 23, numéro 2 (68), hiver 1998

La censure 1920-1960

URI : https://id.erudit.org/iderudit/201368ar

DOI : https://doi.org/10.7202/201368ar

Aller au sommaire du numéro

\section{Éditeur(s)}

Université du Québec à Montréal

\section{ISSN}

0318-9201 (imprimé)

1705-933X (numérique)

Découvrir la revue

\section{Citer ce document}

Luneau, M.-P. \& Hébert, P. (1998). Entrevue avec Fulgence Charpentier. Voix et Images, 23(2), 314-316. https://doi.org/10.7202/201368ar d'utilisation que vous pouvez consulter en ligne.

https://apropos.erudit.org/fr/usagers/politique-dutilisation/ 


\title{
Entrevue avec Fulgence Charpentier
}

\author{
Présentation par Marie-Pier Luneau \\ Entrevue réalisée par Pierre Hébert
}

A priori, la censure en temps de guerre peut apparaitre comme un microcosme idéal pour une étude exhaustive de l'impact censorial sur la société. S'attachant à une période circonscrite dans le temps, elle constitue un ensemble fermé, délimité par des dates très précises (contrairement à la censure religieuse), cercle dont il devient par conséquent possible de faire le tour. Les censeurs sont par ailleurs clairement identifiés et sévissent selon un but obvie (éviter de nuire à l'effort de guerre) en vertu d'articles de lois à première vue nettement moins nébuleux que les règles de morale qui poussent, par exemple, les autorités cléricales à pourfendre un auteur. On pourrait ainsi se demander quelles sont les publications qui, de 1939 à 1945, ont été censurées par l'État et se rengorger en croyant que quelques recherches dans les archives auront tôt fait de répondre à toutes nos interrogations.

Or, le problème est nettement plus complexe. La réponse à la censure, pendant la Seconde Guerre mondiale, se fait au Canada sur un système volontaire. Des comités de censure sont bel et bien mis en place, mais leur fonction n'est que de conseiller les journaux, de leur donner des directives afin qu'ils n'enfreignent pas les Règlements concernant la défense du Canada. Le journal est libre de suivre ou non les directives des censeurs. Le Secrétaire d'État décide, en dernière instance, s'il y a lieu d'intenter des poursuites contre les fautifs. En conséquence, les traces de cette censure devenue autocensure sont rarement visibles.

Par ailleurs, les Règlements concernant la défense du Canada peuvent agir sur deux plans en ce qui concerne la censure. L'article 16 interdit la publication d'informations à caractère militaire, pour éviter que des renseignements confidentiels ne tombent en des mains ennemies; l'article 39A impose une censure de nature politique, en interdisant "[...] particulièrement tout ce qui pourrait causer de la désaffection à l'endroit de Sa Majesté, nuire au succès des Forces alliées, ou nuire au recrutement, à l'entraînement, à la discipline ou à la gouverne des Forces de Sa Majesté ${ }^{1}$ ".

1. Fulgence Charpentier, Paul-André Comeau, Claude Beauregard et Edwidge Munn, La démocratie en veilleuse. Rapport des censeurs 1939-1945, Montréal, Québec/ Amérique, 1995, p. 52. 
L'objet de la censure dépasse alors le cadre des informations militaires pour toucher à des questions politiques nettement plus vastes. Le Devoir, qui respecte l'article 16 mais prend de grandes libertés par rapport à l'article 39A vu ses positions nationalistes, donnera notamment des maux de tête à la censure, comme l'a bien démontré Claude Beauregard dans une thèse récente ${ }^{2}$.

Enfin, pour avoir une idée juste de l'importance du discours censorial pendant la guerre, il faudrait pouvoir mesurer toutes les démarches de la propagande en faveur de l'effort de guerre, forme de censure prescriptive qui tend à vouloir inculquer au sujet une manière de penser, l'orientant vers une perception positive de la participation du Canada au conflit. Les initiatives d'un Claude Mélançon du Service de l'information, qui tente de convaincre Lionel Groulx d'écrire un article en faveur de l'effort de guerre $^{3}$, n'apparaissent-elles pas effectivement comme le pendant "positif " de la censure, puisqu'elle vise à contrôler à la source la parole divergente pour la transformer en discours conforme à l'idéologie dominante? Que dire encore d'un Paul Leduc, directeur dramatique à Radio-Canada qui, à la demande du Service de l'information, suggère à Alfred DesRochers d'établir un lien entre la pièce radiophonique qu'il écrit en l'honneur de Dollard des Ormeaux et la guerre, incitant la jeunesse actuelle à suivre l'exemple du héros du Long-Sault et à s'enrôler pour défendre la patrie menacée ${ }^{4}$ ?

Toutes ces questions mériteraient une étude approfondie qui nous permettrait de brosser un tableau complet de la censure pendant la Seconde Guerre mondiale, tant dans son sens proscriptif que prescriptif. Pourtant, comment retracer l'ensemble de ces démarches puisque, comme nous l'avons noté, la réponse à la censure se fait sur une base volontaire? Les quelques cas qui nous sont parvenus, cas de pressions exercées sur

2. Claude Beauregard, La censure et la guerre. L'expérience des journaux, des militaires et de la population pendant la Deuxième Guerre mondiale, thèse, Université Laval, 1995; id., "Censure et contrôle de l'information au Canada entre 1939 et 1945", Bulletin d'bis-

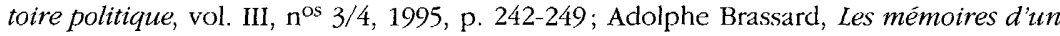
soldat inconnu, Montréal, [s.é.], 1939; Fulgence Charpentier, "La censure en temps de guerre", Revue de l'Université d'Ottawa, 1946, p. 275-300; Paul-André Comeau, Claude Beauregard et Edwidge Munn, op. cit.; Gratien Gélinas et Victor-Lévy Beaulieu, Gratien, Ti-Coq, Fridolin, Bousille et les autres, Montréal, Stanké et Radio-Canada, 1993; Gratien Gélinas et André Fortier, "Gratien Gélinas en entrevue: le théâtre et la censure", Cultures du Canada français, Ottawa, Presses de l'Université d'Ottawa, $\mathrm{n}^{\circ} 8$, automne, 1991, p. 42-43; Renée Legris, Propagande de guerre et nationalismes dans le radio-feuilleton québécois 1932- 1952, Montréal, Fides, 1981 ; Wiliam R. Young, "Le Canada français et l'information publique pendant la Seconde Guerre mondiale ", Bulletin d'bistoire politique, vol. III, $\mathrm{n}^{\text {os }} 3 / 4,1995$, p. 227-242.

3. Voir la correspondance échangée entre Lionel Groulx et Claude Mélançon, Fonds Lionel-Groulx, Centre de recherche Lionel-Groulx, Outremont.

4. Voir la correspondance échangée entre Alfred DesRochers et Paul Leduc, Fonds AlfredDesRochers, Archives nationales du Québec à Sherbrooke. 
des écrivains par le pouvoir censorial, ont été trouvés par hasard dans des fonds d'archives: ils relèvent souvent d'initiatives "non officielles" de la part du personnel du Service de l'information. L'exemple d'Adolphe Brassard, écrivain de Danville qui a dû, suite aux recommandations de Claude Mélançon, retirer du marché son livre Les mémoires d'un soldat inconn $u^{5}$, nous prouve que même si elle s'exerce de façon volontaire, la censure n'en possède pas moins un grand pouvoir: elle a l'avantage de faire pendre au-dessus de la tête des écrivains une épée de Damoclès redoutable.

S'il est donc pour le moment impossible de définir avec précision l'ensemble des démarches du pouvoir censorial auprès des écrivains de l'époque, il n'est pas inintéressant de se tourner vers les témoins de cette période particulière dans l'Histoire. À cet égard, le point de vue de Fulgence Charpentier, censeur pendant toute la Seconde Guerre, revêt une valeur inestimable. Dans un entretien avec Pierre Hébert réalisé à Ottawa le 8 février 1997, il a partagé avec une grande générosité ses souvenirs sur l'époque, permettant surtout de saisir dans quel esprit s'est exercée la censure de guerre.

5. Adolphe Brassard, op. cit. Voir la correspondance échangée entre Claude Mélançon et Bernard Valiquette, Fonds Société des écrivains canadiens, Archives nationales du Québec, BNQ. Nous avons analysé plus à fond les rapports entre Groulx, DesRochers, Brassard et la censure de guerre, à l'occasion d'un colloque sur "L'inscription sociale de l'intellectuel ", tenu à Trois-Rivières, au mois de mars 1997. 


\title{
Entrevue avec M. Fulgence Charpentier
}

\author{
Réalisée par Pierre Hébert à Ottawa, le 8 février 1997
}

V. et I. : M. Charpentier, $\mathfrak{j}$ 'aimerais que vous nous rappeliez dans quel contexte la censure s'est imposée à partir de 1939, compte tenu que vous en étiez responsable du côté francophone.

F. C.: Notez d'abord que ce n'était pas seulement du côté francophone, mais pour tout le Canada. Nous étions deux censeurs qui nous partagions la besogne selon les besoins. J'avais un poste, à cette époque-là, dans l'administration de la Chambre des communes, et j'avais été "prêté" durant la guerre.

V. et I. : Mais dans quelles circonstances vous a-t-on nommé?

F. C. : Lorsque la guerre s'est déclarée, nous, les journalistes - parce que, journaliste, je connaissais le premier ministre et ce qui se passait dans les coûlisses -, nous étions bien placés pour nous occuper d'une question comme celle-là. Deux lois ont été votées: l'une qui concernait l'information, et l'autre pour favoriser l'effort de guerre.

V. et I. : Vous pouvez préciser?

F. C.: Il s'agissait essentiellement de surveiller ce qui se publiait au sujet de la guerre. La censure, c'était d'abord une affaire d'informations militaires; mais elle touchait aussi l'opinion publique, les communications, parce que, comme ça avait commencé à barder, nous étions efficacement préparés. Alors, les autorités ont décidé de mettre sur pied un comité avec, comme chef de la censure, le colonel Biggar. Ce colonel Biggar était un ex-militaire, un avocat francophile d'Ottawa. Mais comme il était avocat, les autorités ont jugé préférable qu'il y ait des "gens du métier", des journalistes. Les journalistes, vous savez, ils ont leur caractère! Des ordres, ils n'en accepteront pas aveuglément. Ils vont se faire donner des conseils, certes, ils vont obéir aux ordres, sans doute, mais il vont exiger d'en comprendre les raisons, car ils devront les expliquer à leurs collègues, qui ne sont pas des soldats, mais des professionnels de l'information. Leur devoir, à ces gens-là, c'est de trouver la nouvelle puis de la sortir. Alors, ils s'exposent...

V. et I. : À des grandes résistances?

F. C.: Oui. Donc, voici ce qui s'est réellement passé. Nos dirigeants ont nommé Claude Mélançon et un journaliste anglais dont je ne me rappelle 
plus le nom. De fait, il n'y avait pas de différence de langue parce que nous faisions le même travail sans partage préalable: les problèmes arrivaient comme ça, jour et nuit, et on jugeait à mesure. Mon collègue anglais pouvait donc faire le même travail que moi.

V. et I. : Mais Claude Mélançon, lui, il était à Montréal?

F. C.: Non. Claude Mélançon avait été représentant de la presse ici, et c'est pour cette raison qu'on lui a confié ce poste. Et c'est pour cela aussi qu'un jour il est parti, pour s'en aller avec Walter Thompson, qui était aussi un de nos collègues journalistes devenu agent de presse. Il est venu me voir et il m'a dit: "Tu sais, je vais être obligé de partir, parce que ça me paye beaucoup plus. Je n'ai pas la formation non plus pour faire ce qu'on va te demander de faire." Il a ajouté : "Il va y avoir une censure et cette censure-là, elle est déjà entre les mains du colonel Biggar. Ce ne sont pas des ordres, mais des directives. Il faut s'entendre avec les journalistes pour qu'ils puissent les suivre."

V. et I. : Des directives, est-ce que cela veut dire que votre pouvoir était passablement limité?

F. C. : Non, pas vraiment. Il s'agissait de dire aux journalistes ce qu'ils devaient taire, en particulier ce qui pouvait renseigner l'ennemi, parce que l'ennemi ne devait évidemment pas tout savoir.

V. et I. : Mais si les directives n'étaient pas suivies, qu'est-ce que vous pouviez faire?

F. C. : Qu'est-ce qu'on pouvait faire? C'est une bonne question. La censure était préventive, mais elle était aussi laissée à la discrétion des journalistes, à cause justement des directives en question. Tous les journalistes disposaient de ces directives, consignées dans un volume qui existe toujours. Or, on n'avait pas vraiment de difficultés, parce que les journalistes ont très bien compris ce qui se passait. Si vous avez affaire à un espion quelque part, allez-vous le rapporter dans votre journal? La loi de la guerre stipule que vous ne pouvez rien dire qui nuirait à l'effort de guerre.

V. et I. : Pourtant, avec Le Devoir en particulier, ça n'a pas été facile?

F. C.: Le Devoir n'essayait pas de renseigner l'ennemi, mais il cherchait plutôt à produire ce qui était contre la guerre. Ses journalistes reproduisaient, très souvent, des articles provenant de journaux suisses. J'avais insulté un jour M. Pelletier, que j'aimais bien d'ailleurs. Je connaissais bien les gens du Devoir, où, jeune, j'avais travaillé. Je connaissais ainsi tous les journalistes, mais celui que je voyais le plus souvent, c'était Georges Pelletier, un ancien avocat. Je l'aimais bien, c'était avec lui que je correspondais, et c'est lui qui m'appelait. Donc, je l'avais insulté un jour en lui disant: "Est-ce que c'est sciemment que vous mettez ça dans la cinquième colonne?" 
V. et I.: Donc vous communiquiez avec lui régulièrement, et pas seulement par lettres. Mais Le Devoir a aussi reçu des lettres...

F. C.: Oui, je communiquais avec lui régulièrement. Mais je n'étais pas seul là-dedans, j'avais des aides, des journalistes professionnels. On avait également des bureaux, cinq en tout, au Canada, qui se trouvaient dans les principales villes, appelées villes "protégées": Halifax, Québec, Montréal... Il y en avait deux dans la province de Québec. À Québec, parce que c'était un gros port de trafic. Il y avait Winnipeg, et aussi la Colombie-Britannique. Là, on avait une vraie équipe; les journalistes ne surveillaient que ce que les journaux locaux écrivaient. Les journalistes devaient obéir aux règlements, et ils savaient tout, autant que nous, bien plus que nous quelquefois, parce qu'il s'y passait beaucoup de choses. Par exemple, les manufactures fabriquaient des pièces de tôle, des moteurs, pour la guerre le plus souvent.

V. et I. : Ainsi, en général, les journalistes obéissaient. Et, comme vous dites, Le Devoir se montrait plus récalcitrant sur certains points.

F. C. : C'étaient des nationalistes. Et les nationalistes disaient, à l'époque, "Ce n'est pas notre guerre".

V. et I.: Est-ce que, ce faisant, les journalistes du Devoir n'allaient pas contre les directives de la censure? Ou, en d'autres mots, est-ce qu'on les a tolérés? Car il y a des journaux qui ont été censurés, qui ont fait l'objet de poursuites judiciaires... Mais pas Le Devoir.

F. C.: Le Devoir a eu des menaces. Des poursuites, il y en a eu, mais rien de majeur. Un incident pouvait arriver et les journalistes le publiaient, sachant qu'il aurait été préférable de le taire. Si on le leur reprochait, ils répondaient: "Mais cela a eu lieu!"Comme M. Pelletier, par exemple, qui déclarait: "Je n'y étais pas cette journée-là, j'avais mal au ventre", ou des choses comme ça. Vous savez, c'était parfois difficile...

V. et I. : Il y avait toujours des faux-fuyants ou des prétextes?

F. C. : Oui, mais ces gens-là n'étaient pas des traîtres, jamais. Cependant, certaines déclarations étaient plus dangereuses, surtout venant de politiciens dont les intentions s'écartaient des nécessités de la guerre. Mais il faut savoir qu'une grande partie de ces ordonnances, de ces directives, touchait des questions de matériel et de présence des personnes beaucoup plus que des déclarations idéologiques. Nous disions constamment aux journalistes: "On ne proscrit pas les idées, mais les déclarations irréfléchies qui renseignent l'ennemi."

V. et I. : Le Devoir se situait plutôt sur le plan des principes, sur le plan des idées que des renseignements factuels?

F. C. : Oui, c'est ça. Dans le cas du Devoir, c'étaient presque toujours des reproductions venant de l'étranger. Des reproductions, même si les journaux ne circulaient pas ici, les journalistes du Devoir en trouvaient, parce 
qu'ils avaient l'esprit tordu, du point de vue de la guerre en tout cas : c'est surtout cela qui nous embêtait. En outre, j'avais des collègues qui lisaient les journaux pour moi. De très bons, qu'on avait pris à La Presse, au Devoir. Des gens comme Willie Chevalier. Alors je lui disais, quand il y avait des choses un peu douteuses: "Prépare-moi donc un rapport, tu as lu tout ça, dis-moi ce que tu en penses. "Il le faisait, mais je ne suivais pas nécessairement sa recommandation. Alors il se fâchait: "Pourquoi me demandez-vous un rapport?" Je lui répondais: "Mon cher ami, ce n'est pas seulement à vous que j'en demande. Il y a plusieurs versions, plusieurs points de vue. Je demande aussi à d'autres leur opinion. "Pour en revenir au Devoir, je vous le dis bien franchement: quand c'étaient les journaux du Québec, on était beaucoup plus tolérants, parce qu'on connaissait leur état d'esprit, et ils n'étaient pas des ennemis, ne voulant tout de même pas la victoire des Allemands! Ils pouvaient dès lors aller loin, mais pas assez pour que ça constitue un crime, ou même un danger. D'ailleurs, nous nous étions entendus avec les journalistes: quand vous entendez quelqu'un qui a de l'influence faire une déclaration intempestive, dangereuse, qui nuit même à l'effort de guerre, demandez-lui si vous pouvez publier sa déclaration avec son nom. S'il y en a qui disent oui, alors publiez-la. C'est contre les règlements, bien entendu, et on doit sévir, mais c'est la responsabilité de l'auteur, pas la mienne. Moi, je suis censeur. En fait, cela est arrivé plusieurs fois. Il y a un cas célèbre, celui du maire de Montréal. Ses propos allaient contre l'effort de guerre, mais on connaissait un peu sa façon de procéder, au maire; et il y a eu toutes sortes d'incidents qui ont ressemblé à l'histoire du maire. Il faut comprendre qu'on était censeurs, ici, de toutes les publications, du livre, de la radio; on voyait les textes de la radio.

V. et I.: Vous les voyiez? Il n'y avait pas de texte qui passait à la radio sans que vous l'ayez vu préalablement?

F. C. : Pas tout à fait. On communiquait plutôt avec la station qui dépendait de nous pour avoir sa permission de diffuser, sachant que violer les règlements l'exposait à se faire couper. C'est pourquoi, dans chaque poste, il y avait quelqu'un qui lisait les textes et disait: "Cela peut passer, cela ne peut pas passer." Mais ça se rendait rarement jusque-là.

V. et I. : "Jusque-là " dans quel sens, vous voulez dire?

F. C. : Jusque-là où il fallait dénoncer une émission à la radio contre l'effort de guerre, non. Dans les cinémas, à cette époque-là, il y avait toujours une comédie, une pièce principale, puis des nouvelles avec des citations des journaux, mais pas nécessairement sur la guerre. Ainsi, quand Mussolini paraissait à la télévision, à Montréal, les gens applaudissaient; c'était de la provocation. Sans nuire vraiment à l'effort de guerre, cette réaction n'encourageait absolument pas l'esprit militaire. Or, quand la situation se produisait à Toronto, qu'on montrait le même film, le même 
personnage, les gens lançaient des briques. C'était un esprit très différent... Alors, nous sommes intervenus: "Quand Mussolini paraîtra, coupez-ça." Ce qui fut fait. N'empêche que la censure s'exerçait souvent sur des choses secondaires comme celle-là, pas sur des grandes questions de trahison!

V. et I. : Mais vous dites qu'il n'y avait rien de majeur.

F. C. : Non. Voici un autre exemple qui a paru dans les journaux. Un jour, à Vancouver, quelqu'un en charge du district militaire, une position d'importance, a déclaré: "Si les Japonais voulaient venir ici, on a tellement peu de défense qu'ils pourraient s'emparer du Canada comme ça!»

V. et I. : Ce n'étaient pas des déclarations à faire...

F. C.: Stupidité! Mais pourquoi le faire? Parce que les militaires aiment exagérer et adorent qu'on leur donne de l'importance. Ils aiment les médailles... Dans son cas, en protestant ainsi comme chef du district militaire, ce militaire donnait une bonne indication aux Japonais pour s'en venir! Mais on savait très bien qu'ils ne viendraient pas, parce qu'ils se faisaient battre là-bas; ils n'étaient pas pour venir se faire battre ici en plus! Ce militaire faisait cette déclaration pour qu'on lui donne d'autres hommes, pour qu'il ait un plus gros personnel sous ses ordres, qu'il soit plus important! À ce propos, les responsables du district militaire m'ont appelé: "Fulgence, pourquoi as-tu laissé passer ça?" J'ai répondu: "J'ai laissé passer ça pour les mêmes raisons qu'avec les journalistes. On ne veut pas empêcher le public de savoir ce qui se passe. On veut empêcher l'ennemi de savoir ce qui est dangereux de lui dire. Entre nous - ce gars-là était colonel -, je pense que certains de vos généraux, ce sont des imbéciles." Alors on me dit: "Fulgence, si ce n'étaient pas des imbéciles, ils ne seraient pas généraux."

V. et I. : Ça venait de clore la discussion!

F. C. : Je voudrais revenir au cas du maire de Montréal, Camillien Houde, qui était un de mes amis en plus. Ce maire savait ce qu'il faisait. Et voici les incidents qui nous poussaient à le croire. Il était élu par une majorité d'Italiens. Vivant dans un quartier italien à Montréal, il savait à qui il avait affaire par ses déclarations. Tout ce qu'il a dit dans une assemblée, le lendemain de la déclaration de la guerre, c'est "Non, ne vous rapportez pas". Les gens disent toujours que c'était pour empêcher la conscription; ce n'était pas la conscription, mais l'inscription. À chaque guerre, il y a un recensement pour connaître les besoins, autant matériels qu'humains. Tous les gens ont dit: "C'est pour savoir combien on a de garçons, pour venir en saisir un." Il y avait autant de déserteurs au Québec, de gens qui se sauvaient dans le bois, qu'il y avait de réfractaires d'esprit, des cas de conscience, des gens qui ne se rapportaient pas, parce que leur foi religieuse ou leur valeurs philosophiques ne leur permettaient pas d'aller tuer d'autres hommes. On ne voulait pas traiter, évidemment, le Québec 
autrement que les autres, mais, de fait, on ne traitait pas ces gens du Québec aussi mal que les autres. Tout de suite, les gens du cabinet nous ont appelés: "Laissez-vous passer ça?" "Je ne peux pas l'empêcher! C'est le maire de Montréal, la plus grosse ville! C'est son opinion! S'il faut sévir, on sévira. Vous ferez ce que vous voudrez. Ça ne change rien pour l'effort de guerre, puisque vous allez vous arranger pour calmer les esprits." On sait ce qui est arrivé [Camillien Houde a été emprisonné], mais seulement ça me faisait de la peine parce que, je le répète, c'était un de mes amis.

V. et I.: Mais de manière générale, l'image que vous me donnez de la censure, finalement, c'est qu'elle ne s'est pas exercée trop durement. Et de votre point de vue, vous n'avez pas eu trop de cas déchirants à traiter. J'aurais pensé le contraire.

F. C.: Il y avait des choses qui nous étonnaient nous-mêmes. On tenait nos assemblées avec le public, c'est-à-dire avec les propriétaires de journaux, chaque année: "Si vous voulez vous plaindre, dites-le-nous." Chaque fois, ils nous ont dit la même chose: "On est heureux de suivre les règlements qui ne nous nuisent pas, qui ne renseignent pas l'ennemi. $\mathrm{Si}$ vous en voulez d'autres, dites-le-nous, on en discutera." Alors, c'était très difficile, à ce moment-là, de faire des recommandations.

V. et I.: J'aimerais aussi que vous nous parliez des éditeurs et des ouvrages littéraires, ou, de manière générale, des productions artistiques ou culturelles. D'abord les éditeurs eux-mêmes: quelles étaient les contraintes ou les types de surveillance auxquels ils devaient faire face? Estce que c'était un peu comme pour les journaux par exemple?

F. C.: Moi, je m'occupais de la presse, et je ne me rappelle pas vraiment, sauf ce qui suit. En France, on publiait des livres qui étaient très antiguerre: les gens de Pétain. Et il y avait des romans aussi. Ces livres-là, on ne les avait pas au Canada. Mais des gens de Montréal y allaient par New York, et les bibliothèques des bateaux avaient ces livres-là. Ils les volaient et les apportaient à Montréal; certains les publiaient. Je ne mentionnerai pas de noms là-dessus. Nous étions ensemble un jour, avec un Français très loquace qui avait passé la guerre ici et qui avait dit: "Vous savez, c'est drôle, ici au Canada, il y a des traîtres et des demi-traitres, comme vous!» Il montrait quelqu'un, il savait que cet homme volait les livres.

V. et I. : Mais les éditeurs eux-mêmes, vous n'aviez pas de contact ou de liens directs avec eux?

F. C. : Non. Je ne connais personne non plus qui l'a proposé... Je l'aurais su.

V. et I. : Adolphe Brassard avait publié un roman, six mois avant la guerre, Les mémoires d'un soldat inconnu; et vous parliez tantôt de M. Claude Mélançon, qui avait justement écrit à Adolphe Brassard, qui habitait Danville à ce moment-là, pour qu'il retire son livre du commerce. C'était un livre qui, même s'il se passait pendant la Première Guerre 
mondiale, était contre l'enrôlement, contre la participation. Mais je ne connais pas d'autres cas...

F. C. : Non, moi non plus, je ne connais pas...

V. et I. : En ce qui concerne la littérature toujours, il y a quand même un auteur québécois qui s'était affiché publiquement contre la censure, Gratien Gélinas, dans ses Fridolinades. Est-ce que vous vous souvenez de ce cas? Car Gélinas avait cette revue-là qui a duré pendant tout le temps de la guerre et qui vraiment allait contre l'effort de guerre dans certains de ses sketches. Et il y a des passages très durs contre l'effort de guerre effectivement, la censure, etc.

F. C.: Je ne me rappelle pas de ça.

V. et I.: Non? Parce que c'est Gratien Gélinas qui dit dans une entrevue qu'un jour, deux agents de la G.R.C. se sont présentés à une de ses représentations pour voir s'il y avait quelque chose de répréhensible, si on peut dire. Et finalement, ils ont dit que non. Mais s'il y avait eu des agents de la G.R.C., est-ce que c'est vous qui les auriez envoyés, à ce momentlà?

F. C. : Oui. Mais ça ne nous a pas été soumis parce que ça ne valait pas le coup, j'en suis certain.

V. et I.: Savez-vous pourquoi ça ne valait pas le coup? Avez-vous une interprétation de ça?

F. C. : On en passait; nos censeurs n'étaient pas si à pic que ça.

V. et I. : Pas aussi acariâtres qu'on pourrait l'imaginer?

F. C.: Non. D'ailleurs, je pense que dans le fond, il s'agissait de ne pas être trop sévères. Ça s'est toujours passé comme ça.

V. et I. : De manière générale, est-ce que ça veut dire, à partir du tableau que vous me donnez, que la censure n'était pas trop mal reçue au Québec? Il n'y a pas eu de protestations importantes? Est-ce que c'est ce que je dois comprendre? Peut-on parler de censure douce?

F. C. : En ce qui concerne $80 \%$ de la censure, certainement, c'étaient des affaires insignifiantes.

V. et I. : Et en 1946, si je me rappelle bien, vous avez vous-même rédigé un article qui est paru dans la Revue de l'Université d'Ottawa, et qui fait un peu le tour de la question de la censure durant la guerre. Qu'est-ce qui vous a amené à rédiger ce texte? Est-ce que c'était une initiative personnelle?

F. C. : C'étaient les gens de l'Université.

V. et I. : Qui vous ont commandé cet article-là, si on peut dire?

F. C.: Commandé, non. Ils m'ont demandé: "Si vous vouliez parler de la censure dans le prochain numéro, il y aurait de la place." 
V. et I. : Et vous vous y êtes prêté de bon gré?

F. C.: Oui, pourquoi pas? Ce n'est pas défendu d'écrire.

V. et I. : Non, même sur la censure!

F. C.: Même sur la censure! Mais voici. Quand la guerre s'est terminée, il a fallu attendre que le Japon se redresse. Je suis allé un jour en France, mais la guerre n'était pas finie. Il y a eu comme un flottement à la fin de la guerre entre la libération de Paris et la chute des Japonais, de plusieurs mois (quand je dis plusieurs mois, je crois que c'était trois ou quatre mois, assez longtemps). En tout cas, j'étais là-bas pour organiser la censure en Allemagne. À ce moment-là, la question de la censure est remontée à la surface et j'ai dit au ministre: "Voici la situation. Nous avons ramassé cette information-là, elle a été communiquée à ceux qu'il fallait, l'armée en particulier; tous ces rapports, qu'est-ce qu'on en fait maintenant?" Je dois vous dire qu'on en avait six millions. Ça ne sert plus à rien, la guerre est finie. Seulement, c'est de l'information qu'on a recueillie, en vertu d'une loi. Cette loi-là, elle n'existe plus, donc on n'a plus le droit d'avoir l'information! Alors on va préparer un rapport, dire comment ça s'est passé, dire ce qu'on a fait. Parce que tous les types qui prennent le poste d'officier dans l'armée sont obligés de le lire, pour savoir comment organiser ça rapidement. Vous savez, la vie c'est une affaire imprévue, il faut être prêt.

V. et I. : Alors ça, c'est votre fameux rapport.

F. C. : Fameux, oui, c'est une façon de parler... En tout cas, le rapport est sorti. J'ai dit au ministre: "Je vais vous donner mon rapport, tout l'essentiel sera dedans, les recommandations, comment ça a marché. Seulement il y a les papiers. On n'est pas pour se promener avec les papiers..." $\mathrm{Ce}$ fut recueilli, ramassé, encore une fois secrètement, en vertu d'une loi qui disparaît.

V. et I. : Alors vous aviez tous ces rapports.

F. C.: Oui, on avait tous ces rapports; alors j'ai dit, voici ce qu'on va faire: tout ce qui se rapporte à des crimes, on va le donner à la police. Puis, il y a tout le reste, des affaires insignifiantes, secondaires et beaucoup de choses indiscrètes se rapportant à la guerre, toujours obtenues en vertu d'un règlement désuet. Ce serait mieux de les faire disparaître; on va les brûler. Le ministre me dit: "D'accord. C'est mieux comme ça."

V. et I.: J'aurais une dernière question à vous poser. Si on était aujourd'hui en 1939, et qu'on vous demandait de vous occuper de la censure, est-ce que vous accepteriez à nouveau?

F. C.: Je ne sais pas. Belle question. Il faudrait que j'y pense à deux fois. Parce que vous savez, il y a beaucoup de choses (bien que ça n'ait pas changé ma vie, mais...) qui ne sont pas pareilles, quand même. Je crois 
qu'il y aurait des règlements de censure, parce qu'il y a des renseignements qu'il ne faut pas divulguer, surtout dans le domaine industriel. L'histoire des idées, c'est une autre affaire: là, un homme est libre. Mais il faut des règlements. Si quelqu'un dit le contraire de l'opinion publique, ça peut aller mal. À ce moment-là, il n'y a pas que la censure qui se mêle de ça: quand quelqu'un fait des déclarations publiques, n'importe qui peut les rapporter, pas seulement les journalistes. De plus, il y a des gens qui sont contre l'effort de guerre tout le temps: ils sont carrément contre la guerre.

Il y a des erreurs aussi dans le monde. On me demande souvent, à cause de mon âge invraisemblable ${ }^{6}$ : "Est-ce que vous trouvez que les gens vivent mieux aujourd'hui qu'il y a cent ans?" Je crois qu'ils vivent mieux, que la vie est plus facile. C'est plus facile d'aller chercher de quoi manger de l'autre côté de la rue que de courir des milles, de se déplacer, de tout avoir. Dans le temps de nos grands-pères, ce n'était pas pareil. Mais comment se fait-il qu'avec une vie plus facile, il y a des enfants qui se suicident? Il y a quelque chose qui va mal quelque part. Comment se fait-il que, plus la vie est facile, moins les gens endurent la vie?

V. et I. : Qu'est-ce que vous en pensez? Avez-vous des éléments de réflexion ou des réponses à ces questions importantes?

F. C. : Ce sont des choses qui me troublent.

V. et I. : Et on est comme sans réponse...

F. C.: On ne réussit jamais à avoir réponse à tout. Il faut expliquer tout ça. En avez-vous, une bonne explication, vous? On se dit, j'espère qu'il y a un ciel pour qu'il y ait une justice quelque part. Qu'est-ce que ça va être, la justice, quelque part? En admettant qu'il va y en avoir une... Les gens qui se rencontrent, à des siècles de distance, de quelle façon vont-ils raisonner? Et s'il y a un bon système, qu'est-ce que c'est? Où est-il, le bon système? Est-ce qu'il existe? Il y a toutes sortes de savants qui ont pensé à tout ça, depuis Socrate, et on cherche encore! C'est un peu cela que vous me demandez... En tout cas, je trouve tout simplement que si on travaille pour s'entraider, le monde va mieux marcher.

6. En juin 1997, M. Charpentier fêtait son centième anniversaire de naissance. 